Article

\title{
Assessing Global Forest Land-Use Change by Object-Based Image Analysis
}

\author{
Erik J. Lindquist ${ }^{*}{ }^{\dagger}$ and Rémi $D^{\prime}$ Annunzio ${ }^{\dagger}$ \\ United Nations Food and Agriculture Organization, Rome 00153, Italy; Remi.Dannunzio@fao.org \\ * Correspondence: erik.lindquist@fao.org; Tel.: +39-065-705-2875 \\ + These authors contributed equally to this work.
}

Academic Editors: Ioannis Gitas, Clement Atzberger and Prasad S. Thenkabail

Received: 4 March 2016; Accepted: 15 August 2016; Published: 20 August 2016

\begin{abstract}
Consistent estimates of forest land-use and change over time are important for understanding and managing human activities on the Earth's surface, parameterizing models used for global and regional climate change analyses and a critical component of reporting requirements faced by countries as part of the international effort to Reduce Emissions from Deforestation and Degradation (REDD). In this study, object-based image analysis methods were applied to a global sample of Landsat imagery from years 1990, 2000 and 2005 to produce a land cover classification suitable for expert human review, revision and translation into forest and non-forest land use classes. We describe and analyse here the derivation and application of an automated, multi-date image segmentation, neural network classification method and independent, automated change detection procedure to all sample sites. The automated results were compared against expert human interpretation and found to have an overall agreement of $\sim 76 \%$ for a 5-class land cover classification and $\sim 88 \%$ agreement for change/no-change assessment. The establishment of a 5 ha minimum mapping unit affected the ability of the segmentation methods to detect small or irregularly-shaped land cover change and, combined with aggregation rules that favour forest, added bias to the automated results. However, the OBIA methods provided an efficient means of processing over 11,000 sample sites, 33,000 Landsat $20 \times 20 \mathrm{~km}$ sample tiles and more than 6.5 million individual polygons over three epochs and adequately facilitated human expert review, revision and conversion to a global forest land-use product.
\end{abstract}

Keywords: Landsat; land-use; land cover; OBIA; segmentation; change detection; sample; minimum mapping unit

\section{Introduction}

In an effort to produce a set of spatially consistent and comparable statistics on global tree cover, forest area and change, the United Nations Food and Agriculture Organization (FAO) in collaboration with the Joint Research Centre of the European Commission (JRC) used object-based image analysis (OBIA) techniques and remotely sensed satellite imagery to implement a sample-based survey of the Earth's land surface called the Global Forest Resource Assessment (FRA) 2010 Remote Sensing Survey. Forest land-use change was estimated at global, regional and ecological domain scales for the time period 1990-2005 [1].

OBIA is increasingly used to classify remotely sensed data [2] in a process that includes image segmentation techniques as an integral part of the classification. Image segmentation is the process of combining the individual picture elements (pixels) of raster data into meaningful objects for identification purposes [3]. Merging pixels of similar spectral and proximal spatial properties together and assigning a common label accomplish this. 
Object-based methods have been frequently used in land cover classification. Classic methods of achieving this result include the unsupervised clustering algorithms of ISODATA [4] or k-means [5], in which pixels with similar spectral qualities are assigned a common label depending on rules specified by the user including the total number of classes desired in the output image. Though segmentation algorithms have advanced since, the basic premise remains the same: to delineate areas on the Earth's surface meaningful to the purposes of analysis. OBIA methods extend the classic pixel clustering algorithms by also incorporating shape and other characteristics of the pixel clusters formed during image segmentation. Hussain et al. [6] provide a thorough review of the advantages of OBIA and change detection methods and applications.

OBIA approaches have recently been applied in a number of studies to successfully classify land cover and land use. Viera et al. [7] used image segmentation and data mining algorithms to successfully classify patterns of agriculture in Brazil. Eva et al. [8,9] used image segmentation techniques to assess forest cover, forest cover change and associated carbon dioxide emissions in South America. Duveiller et al. [10] also used image segmentation of medium spatial resolution imagery to classify land cover and change for a sample-based assessment in the Congo Basin. Ernst et al. [11] also assessed forest cover and change for the Congo Basin using medium spatial resolution data. Mayaux et al. [12] describe the results of a forest cover change assessment, also for the humid tropics of Central Africa, obtained from a sample-based assessment of segmented medium resolution imagery. Rasi et al. [13] describe the use of OBIA techniques for classifying medium spatial resolution imagery pan-tropically. Brink et al. [14] describe the results of a land cover change detection for parts of Africa over 25 years using OBIA methods. Bodart et al. [15] estimated tree cover change in dry Africa from 1990 to 2000 using OBIA and medium spatial resolution imagery.

Image segmentation of medium spatial resolution imagery for the purposes of delineating forest cover and forest cover changes, however, may be subject to problems with spatial scale and associated effects on detectability of change [16]. Landsat pixels represent an arbitrary grid with $30 \times 30 \mathrm{~m}$ cells in which spectral reflectance from the surface of the Earth is recorded. Thus, pixels by themselves are not necessarily optimally placed to represent identifiable objects $[17,18]$ and, depending on the spatial resolution of the input satellite imagery, a single pixel can represent anything from a portion of a tree crown (e.g., for very fine spatial resolution imagery) to many trees (e.g., for medium spatial resolution imagery) to entire stands or stand complexes (e.g., for coarse spatial resolution imagery).

Within-class spectral heterogeneity leads to difficulties in both land cover classification and change detection [16,17]. Ideally, image segmentation will minimize spectral heterogeneity within objects. However, as object size increases so too does heterogeneity; large objects from a segmentation will likely be more heterogeneous than small objects [3]. In the case of detecting forest cover changes in Landsat imagery using segmentation techniques, it may be that the scale of the change occurs at finer levels than the minimum size of the image segment, especially if the segments are coerced to an arbitrary minimum mapping unit.

This paper describes and analyses the OBIA methodology used by the FAO to classify land cover and land use for over 11,000 globally distributed, satellite image-based sample sites. We provide an analysis of the image segmentation and classification and we revisit the problem of scale in satellite image classification [16], namely the selection of a minimum mapping unit, its potential addition of bias to the results and the effectiveness of image segments coerced to a pre-defined minimum mapping unit at detecting land cover changes of different shapes and sizes when using medium spatial resolution imagery. Finally, we examine the differences between tree cover and forest land-use, the practicalities of classifying both and the difference in global forest area when they are considered separately.

\section{Materials and Methods}

The United States Geological Survey's (USGS) Global Land Survey dataset (GLS) was used as input imagery in this assessment. The GLS is a geometrically corrected dataset that covers most of the Earth's land surface and is composed of the single best Landsat- 4 TM, -5 TM and 
-7 ETM+ image acquisition centred on the years 1990, 2000, 2005 and 2010 [19]. The GLS acquisitions were radiometrically normalized to improve the performance of automated classification and change detection algorithms [20-22]. This study considers GLS data from 1990, 2000 and 2005 only.

A sampling design with a site at each $1^{\circ}$ intersection of latitude and longitude was employed, except in Canada and the Russian Federation (see [1] for details). Sampling intensity was reduced above $60^{\circ}$ latitude north and south to include only even degrees of longitude. No sample sites were located higher than $75^{\circ}$ north or south latitude. At each sample site, Landsat $30 \times 30 \mathrm{~m}$ optical bands 1-5 and 7 were subset to a central $20 \mathrm{~km}$ by $20 \mathrm{~km}$ box [21,23]. The GLS data were assumed to be the best data available for each site. If more than one GLS acquisition was available for a given site and date the GLS acquisition with the least cloud cover was selected for classification.

The JRC, as part of its on-going Tropical Ecosystem Environment Monitoring by Satellites or TREES [24] and FOREST [25] forest monitoring programmes, processed approximately 4700 pan-tropical and western European survey sites, respectively [13]. The FAO processed sites located in the sub-tropical, temperate and boreal regions of the Americas, Asia, Europe including Russia and Oceania (Figure 1). The entire global sample grid of 15,770 sites is equivalent to a $1 \%$ sample of the Earth's land surface.

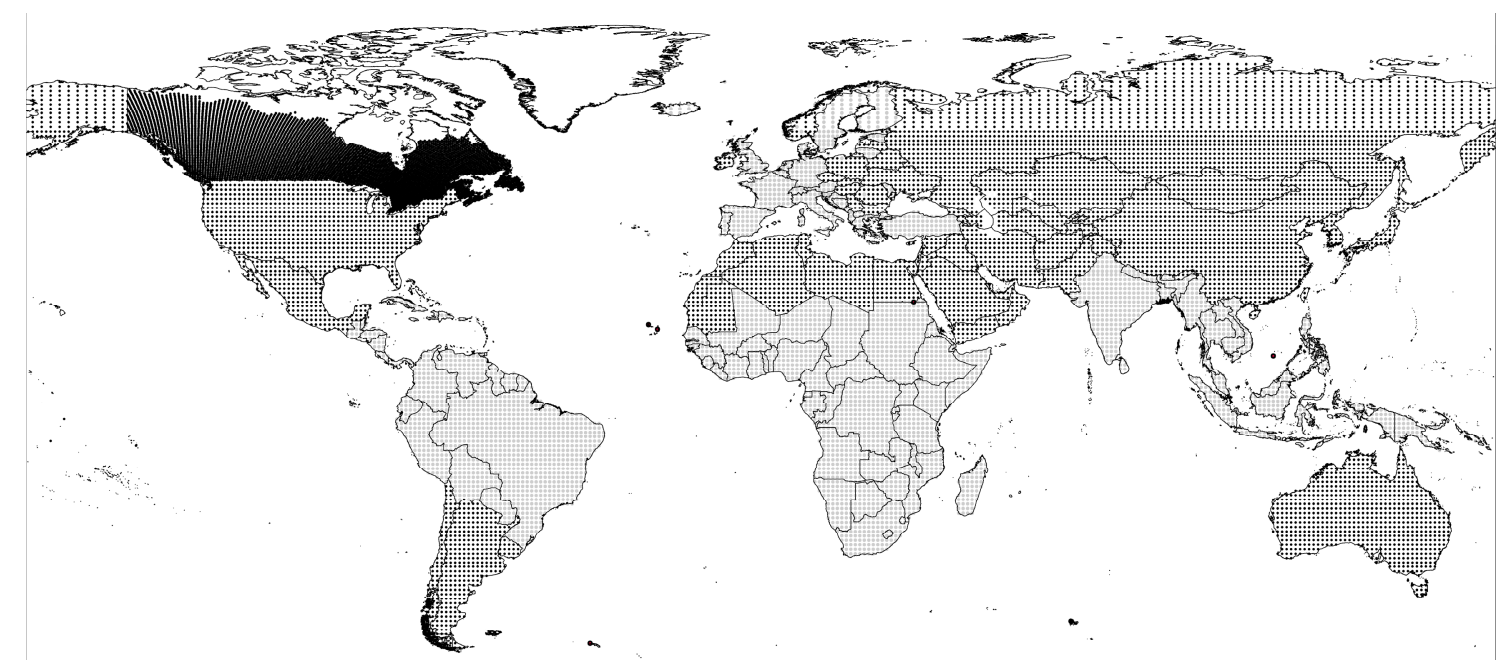

Figure 1. The global, degree grid framework, including the Canadian NFI plot scheme. FAO processing methodology was applied to sites $(>11,000)$ in the boreal, temperate and sub-tropical climatic domains (in black) (From [1]).

\subsection{Image Segmentation}

Image segmentation is the process of partitioning an image by grouping pixels into clusters, called objects, based on intra-object spectral similarity and inter-object spectral differences. In this study, a region-growing multi-resolution image segmentation algorithm was used in which the criteria for creating image objects from individual pixels can be adjusted by specifying values for a series of parameters that control: (i) object average size, called the scale factor; (ii) object spectral homogeneity, called the shape factor; and (iii) object boundaries, called the compactness factor [26].

Landsat image bands 3, 4 and $5(0.63-1.75 \mu \mathrm{m})$ from Landsat-4 TM, -5 TM and -7 ETM+ and all three GLS epochs were used in a multi-date segmentation routine to create segments capturing spatially contiguous areas of similar spectral response and areas with unique temporal spectral signatures (Figure 2) [27]. These particular Landsat bands were selected for their ability to discriminate differences in surface reflectance caused by changes in vegetation cover [10,28]. A parsimonious selection of image bands for segmentation generally improves the quality of the segmentation and reduces the chances of over-segmentation (e.g., creating many more segments than are necessary to define objects of interest) [28]. 

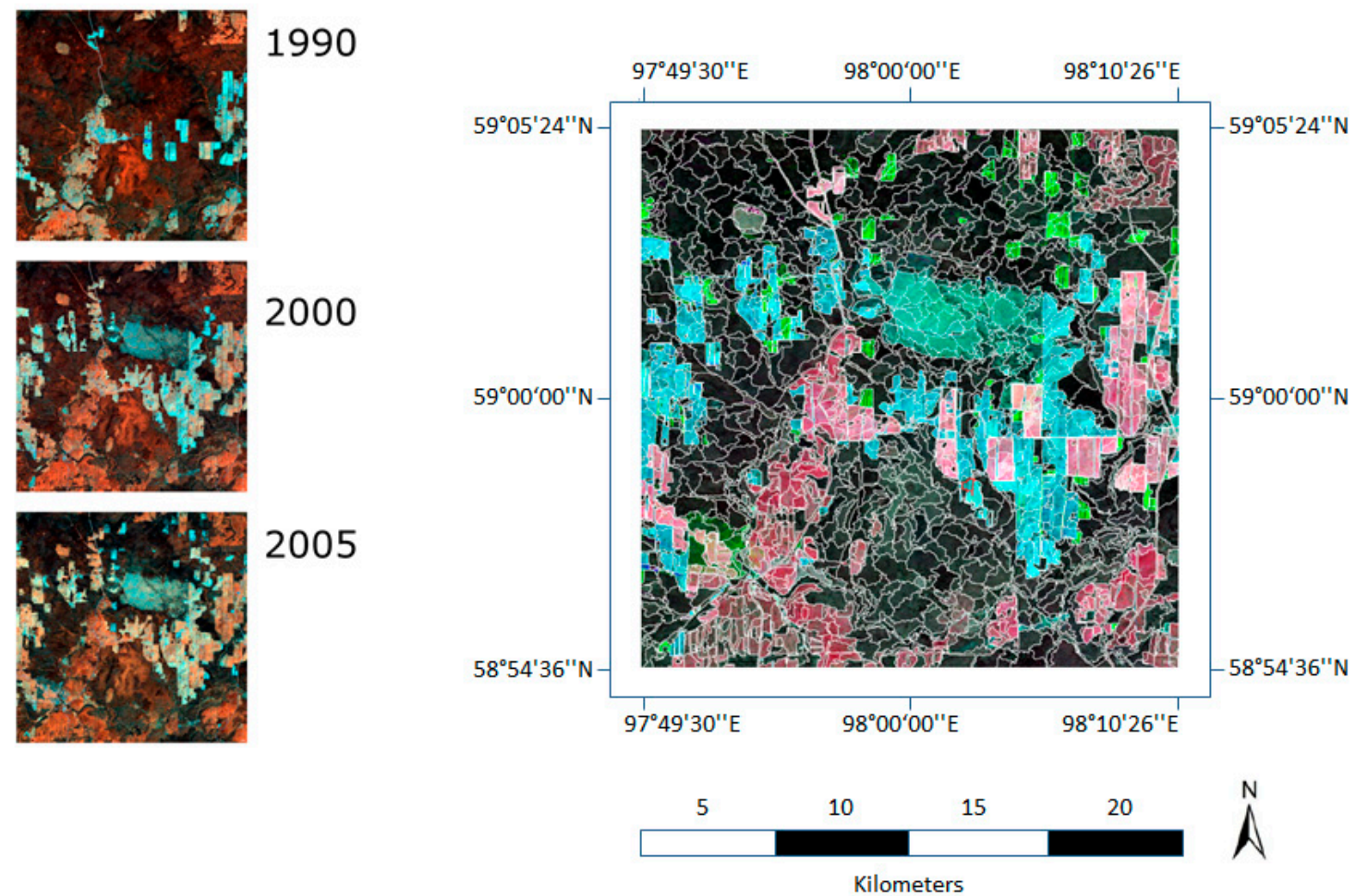

Figure 2. (From [1]) A multi-date segmentation for a $20 \mathrm{~km} \times 20 \mathrm{~km}$ site in the boreal climatic domain. Landsat imagery from 1990, 2000, 2005 are combined into a single data stack and segmented. The segments (white outlines in image on right) capture areas of spectral similarity as well as areas of change over time. The Landsat image on the right is a colour composite of Landsat band 5 from 1990 (red), 2000 (blue) and 2005 (green). Changes in tree cover between time periods are evident.

Image segmentation was a two-step process, defined in this paper as level- 1 and level-2. The same level-1 segmentation parameters (scale $=15$, shape $=0.1$, and compactness $=0.5$ ) were fixed for all sample sites as no a priori information about the sample sites was assumed. These parameters were selected to create relatively fine-scale image objects (scale $=15$ ) with no preference towards "compactness" (compactness $=0.5$ ) and with object "colour" being one of the most important criterion (shape $=0.1$ ) defining object homogeneity. Since the goal of the study was global forest change, no specific effort was made to optimize the segmentation for any particular locale but, rather, to have a consistent approach globally that effectively partitioned image pixels into homogenous segments that could be combined at the desired minimum mapping unit (MMU). Level-2 objects were created to meet a MMU requirement of five hectares. This MMU was chosen to allow resolution of relatively small forest cover changes and maintain a manageable number of image segments [29]. To meet the MMU requirement, level-1 segments less than five hectares in size were merged with adjacent objects with the most similar average Landsat band 5 short-wave infrared reflectance $[30,31]$ until the minimum size requirement was met.

\subsection{Automated Image Classification}

For each site and date, a land-cover classification was produced with five classes: tree cover, other wooded land, other land, water and no data. Tree cover was defined by woody vegetation greater than five meters in height. Other wooded land cover was defined as woody vegetation less than five meters in height. Other land cover was defined as all other land cover types, including herbaceous and bare land. Clouds, cloud shadow and missing data were given a "no data" label. Figure 3 illustrates the processing method applied to a sample site. 
Level-1 image objects were classified first for year 2000 using a supervised approach with training data collected from the year 2000 Landsat data. Training data for all land cover classes were selected automatically utilizing temporally and spatially coincident land cover data from the Moderate Resolution Imaging Spectroradiometer (MODIS) Vegetation Continuous Fields (VCF) product [32], the year 2005 GLOBCOVER global land-cover product [33] and the MODIS global $250 \mathrm{~m}$ water mask [34].

A set of potential training objects were identified from all possible level- 1 image objects by first flagging those most likely to represent either unambiguous tree cover or unambiguous non-tree cover (e.g., other land). Per-object class homogeneity was defined in relation to the range of VCF percent canopy-cover values circumscribed by the object (Figure 3B). An initial VCF range threshold value of 10 (e.g., Max VCF - Min VCF < 10) was chosen. To ensure the availability of ample training data, if less than $30 \%$ of the total number of image objects were flagged using the initial VCF threshold, the threshold was increased by a value of five and the selection process repeated. Iterations continued until at least $30 \%$ of the total image objects were identified as the potential training dataset.
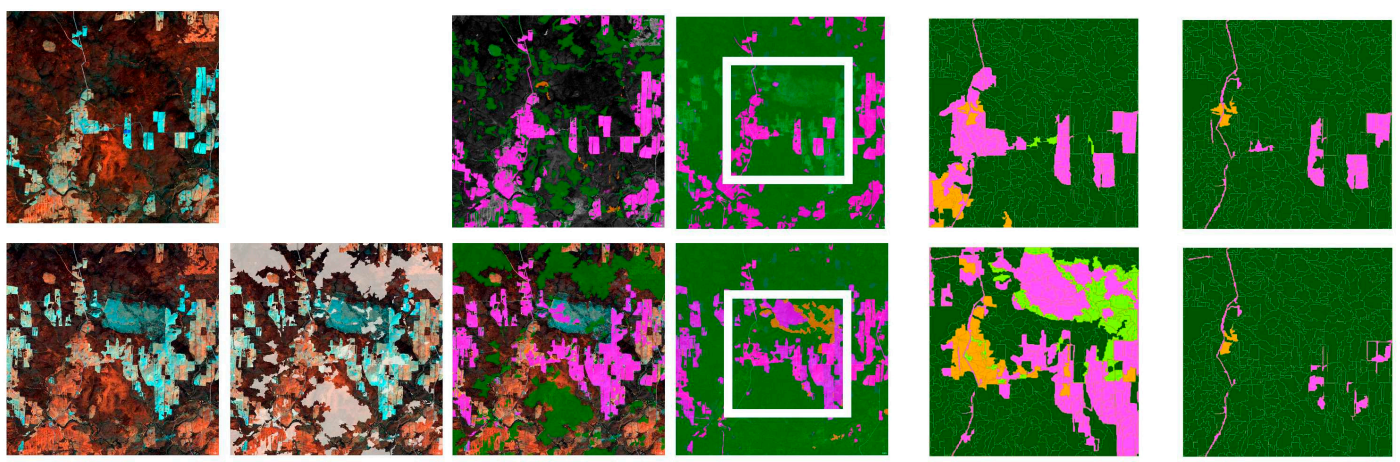

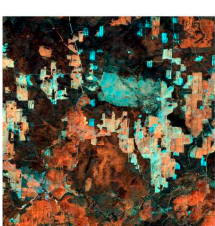

A

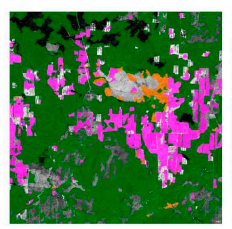

C

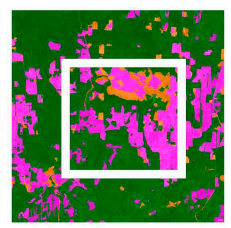

D

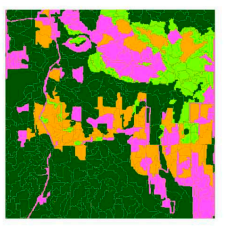

$\mathrm{E}$

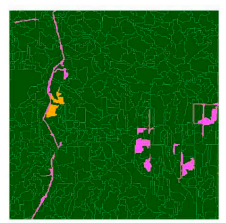

$\mathrm{F}$

Figure 3. The multi-date processing scheme over the same area as in Figure 2 for year 1990 (top row), year 2000 (middle row) and year 2005 (bottom row) the following: (A) the $20 \times 20 \mathrm{~km}$ Landsat imagery in colour composite b3,4,5; (B) the potential training objects (grey) for year 2000; (C) the training dataset after class labelling, where Dark green $=$ Tree Cover, Orange $=$ Other Wooded Land, and Pink = Herbaceous; (D) the final automated land cover classification, where Dark green = Tree Cover, Orange = Other Wooded Land, and Pink = Herbaceous; $($ E) the final, expert revised land cover classification of the central $10 \times 10 \mathrm{~km}$ (corresponding to white box in D), where Dark green = Tree Cover, Light green $=$ Tree Cover Mosaic, Orange $=$ Other Wooded Land, and Pink = Herbaceous; and $(\mathbf{F})$ the corresponding final, expert revised land use classification, where Dark green = Forest, Orange $=$ Other Wooded Land , and Pink $=$ Other Land Use.

Next, class labels were assigned to the training dataset using a simple rule set consisting of a combination of 2000 MODIS VCF, 2005 GLOBCOVER, MODIS Surface Water Bodies land-cover products and hard-coded Landsat digital number (DN) thresholds (Table 1 and Figure 3C). Segments indicated as bare ground or cloud cover were labelled as such and removed from the training data set. Clouds were hard-coded as no data and bare ground was hard-coded as other land in the final land cover classification. 
Table 1. Training segment labelling rules based on underlying MODIS VCF, GLOBCOVER, MODIS Water Mask and hard-coded Landsat DN thresholds. Landsat bands refer to those of Landsat- $4,-5$ TM and -7 ETM+.

\begin{tabular}{ll}
\hline Land Cover Class & Segment Characteristic \\
\hline Water & Segment proportion of Global Water Mask $>0.7$ \\
\hline Tree & Mean segment VCF\% tree cover $>11$ \\
\hline Other Wooded Land & Mean segment VCF\% tree cover $<11$ AND Globcover Class = Shrub \\
\hline Herbaceous & $\begin{array}{l}\text { Mean segment VCF\% tree cover }<11 \text { AND Globcover Class }=\text { Herbaceous } \\
\text { ORLandsat Band 5 DN }>120\end{array}$ \\
\hline Bare ground & Mean segment Landsat Band 2 DN $>200$ AND Landsat Band 5 DN $>200$ \\
\hline Cloud & Mean segment Landsat Band 4 DN + Band 5 DN + Band 7 DN $>500$ \\
\hline
\end{tabular}

\subsection{Neural Network Land Cover Classification}

An artificial neural network (ANN) classifier was used to create the final land-cover classification. Neural networks consist of input layers, a layer of processing nodes, referred to as the "hidden layer", and output layers. The generalization power of neural networks is well suited for supervised vegetation classification with non-parametric data and sub-optimal training [35-37]. A neural network classifier was chosen over other possible classification algorithms due to their generally good performance in vegetation classification [36] and the ease of use within the eCognition software. A simple feed-forward ANN with back-propagation is implemented within eCognition through the Neural Network Plugin from Freiberg University [38].

Classification inputs came from the selected training segments and consisted of the segment class label, the mean segment digital number (DN) of Landsat image bands $3(0.63-0.69 \mu \mathrm{m}), 4(0.77-0.90 \mu \mathrm{m})$, $5(1.55-1.75 \mu \mathrm{m})$ and $7(2.09-2.35 \mu \mathrm{m})$ and, finally, all possible simple 2-band ratios. A simple ratio of Landsat bands $2(0.52-0.60 \mu \mathrm{m})$ and $7(2.09-2.35 \mu \mathrm{m})$ was also included, making a total of 11 input layers. Band ratios were used to improve discrimination of forest, non-forest and deforestation. Hansen et al. [39] used simple 2-band ratios to aid classification of forest cover and deforestation in Central Africa. The number of nodes in the hidden layer was arrived at empirically and numbered 16.

The ANN was applied sequentially to the year 2000 image objects to create a series of "masks" to separate major land cover classes from each other and refine the classification as the sequence progressed. The first ANN was applied to the image to distinguish water from land. The "land" class, and only the "land" class, was then divided into herbaceous vegetation and woody vegetation. The woody vegetation objects were split into tree cover and other wooded land classes. In each model, two output nodes were established, corresponding to the number of desired land-cover classes (e.g., water or other, herbaceous or woody, tree or other wooded land). Training segments were relabelled in each iteration to correspond to the desired output. For example, in the first division between water and land, all non-water training data were relabelled as "land" and the ANN was run. The same was done for each successive iteration. Each time the ANN was allowed to train itself 1000 times per class using a back-propagation learning method. Once the training of the network was complete (i.e., when the error was minimized or the 1000 training runs completed) the model was applied to all level-1 image objects within the scene.

ANN performance can be affected by the quality and amount of data used to train the model $[37,40]$. To ensure neither over- nor under-training the model, the total number of training observations per site was restricted to approximately 2000 (e.g., roughly half the total average number of level-1 image segments per sample). Each training class was reduced and harmonized by proportional random sampling until the total number of samples reached the total allowable number of training observations. If a training class had less than 100 observations to begin with, all observations were used to construct the final model. 


\subsection{Multivariate Alteration Detection for Land-Cover Change}

Change between time periods was assessed independently from the land cover classification by employing the iteratively re-weighted multivariate alteration detection (IR-MAD) algorithm [41-43]. The IR-MAD algorithm is an extension of canonical correlation analysis that maximizes the information on change over all variables (i.e., satellite image bands) considered in the analysis. The algorithm is insensitive to spectral changes that occur between co-located, bi-temporal data due to changing sensor characteristics like gain and bias, linear data normalization or calibration and affine transformations. IR-MAD improves on previous multivariate change detection algorithms [41] by iteratively increasing the weight of non-change observations and theoretically improving the discrimination of substantive spectral changes between time periods. IR-MAD was implemented within the eCognition 8.0 software as the MAD-Transformation plugin [44].

Landsat bands $3(0.63-0.69 \mu \mathrm{m}), 4(0.77-0.90 \mu \mathrm{m}), 5(1.55-1.75 \mu \mathrm{m})$ were used in an IR-MAD analysis to label image objects with spectral changes between time periods. The first order MAD variate was computed from the selected Landsat bands and their differences between 1990-2000 and 2000-2005. Image objects in 1990 and 2005 more than 0.25 standard deviations away from the overall site-wide mean for the MAD variate were flagged as having a high likelihood of land cover change between the survey periods.

\subsection{Assigning Class Labels to 1990 and 2005 Time Periods}

Land cover labels were assigned to 1990 and 2005 image objects based on whether or not the object represented a potential land cover change. Unchanging 1990 and 2005 image objects were directly assigned the land cover label from year 2000. Year 1990 and 2005 image objects exhibiting a likely change in land-cover, relative to year 2000, were assigned a land cover class by supervised classification using training data taken from the unchanged image objects from each time period, respectively (Figure 3C). Training set reduction and ANN application were as for year 2000.

Level-2 image objects (with a five-hectare MMU) were assigned land-cover labels based on the underlying level-1 segments according to the rules in Table 2.

Table 2. Level-2 segment labelling rules for the automated land cover classification. Level-2 labels were based on the areal proportion of component level-2 segments.

\begin{tabular}{ccc}
\hline Level 1 LC & Percent Composition & Level-2 LC \\
\hline Tree cover & $\geq 30$ & Tree cover \\
Other wooded land & $\geq 70$ & Other wooded land \\
Other land cover & $\geq 70$ & Other land cover \\
Water & $\geq 70$ & Water \\
\hline
\end{tabular}

The final land cover classification for the central $10 \times 10 \mathrm{~km}$ portion of each sample was subject to manual expert review and revision by image interpreters with local knowledge to correct classification errors caused by the automated procedure (Figure 3D). The final, revised land cover labels (Figure 3E) were ultimately converted to land use categories and the experts were again employed to modify the land use labels where necessary (Figure 3F) (see Lindquist et al. [1] for conversion details).

\subsection{Land Cover vs. Land Use}

Land cover refers to the biophysical attributes of the Earth's surface and can be detected directly from aerial imagery or satellite-borne sensors. Land cover describes what is on the ground at a given moment. Land use, on the other hand, implies a human dimension or purpose for which the land is used [45]. The FAO FRA forest definition is land use based so the automatic classification of satellite imagery into land cover information needed to be finally interpreted into land use to match this requirement. 
The conversion from land-cover class to land-use class is a two-step process and required the input of expert human interpretation. First the land-cover classes were automatically converted into one of four land-use classes: (i) forest; (ii) other land use with tree cover; (iii) other wooded land; and (iv) other land use. For example, all objects with the "tree cover" land cover were automatically relabelled as "forest" land use. The other land cover classes were also translated directly to land use but their class label remained the same. This direct translation into land use classes correctly accounted for a large proportion of the objects within the sample sites. Accurate labelling of land use, however, must be examined in a functional and ecological context that includes determining not only what human activity is taking place and what vegetation is there at the time of the satellite image acquisition but also how that land area will respond in the future (e.g., through regeneration, afforestation, or permanent deforestation) [46]. Thus, many exceptions to the direct and general rules concerning land cover to land use transitions exist. To account for these exceptions, land-use labels were re-coded manually wherever these exceptions were present.

Tree land cover corresponds to forest land use, except when the predominant land use is not forestry. This includes, for example, all urban areas, orchards, oil palm plantations, agricultural land with trees and areas under agroforestry. Such lands are classed as other land-use with tree cover.

Other wooded land cover corresponds to other wooded land use, except when the object represents tree cover regeneration in which the woody vegetation will ultimately achieve height and density requirements to meet the definition of forest.

Other land-cover corresponds to other land-use except when it is a forest stand which is cleared and prone to regenerate or to be replanted, in which the object is relabelled as forest land-use.

The review and revision of the land use classification was aided by freely available very-high-resolution satellite imagery. For each sampling tile of the design, the segments were converted into KML format and overlaid into Google Earth ${ }^{\mathrm{TM}}$, together with images from the Degree Confluence Project, Panoramio ${ }^{\mathrm{TM}}$ and existing vegetation maps, where available. Specific expert field knowledge proved very important to better understand the context and interpretation of the landscape.

\section{Results and Discussion}

\subsection{OBIA, Minimum Mapping Units and Land Cover Classification}

The segmentation routine and enforcement of a 5 ha MMU represents a trade-off between segment homogeneity, data volume and ease of use by expert image interpreters [2,3]. However, increasing the heterogeneity of image segments to meet an a priori MMU can make classification difficult and introduce bias in the estimates of area and area changed.

A sub-sample of sites was analysed to explore the effect of the segmentation routine on object size and data volume. The initial, level-1 segmentation produced image objects from $900 \mathrm{sq}$. m. (a single pixel) to $>125$ ha in size. The mean area of level-1 polygons was 11.8 ha. The level-2 segmentation, with a MMU of 5 ha, had a mean polygon size of 17 ha. The merging of level- 1 to level- 2 polygons did not change the relative size distribution, with a large portion of the polygons remaining $<15$ ha in size. The level-2 segmentation, in which small, spectrally homogenous areas were merged with larger, neighbouring polygons, decreased the total number of polygons by $30 \%$.

Increasing the size of the image objects to meet the MMU combined with the MMU segment labelling rules in which any level-2 object containing $>29 \%$ tree cover resulted in a small bias amounting to a $3 \%$ increase in estimated area of tree cover when compared against the level-1 segment results. This bias was removed after the expert review and revision, however the expert review increased the total area of tree cover by $3.5 \%$ over the initial level- 1 classification. In this sub-sample, expert revision revealed that the automated algorithm produced a slight overestimation of tree cover where tree cover is low and a slight underestimation of tree cover where tree cover is high (Figure 4). 

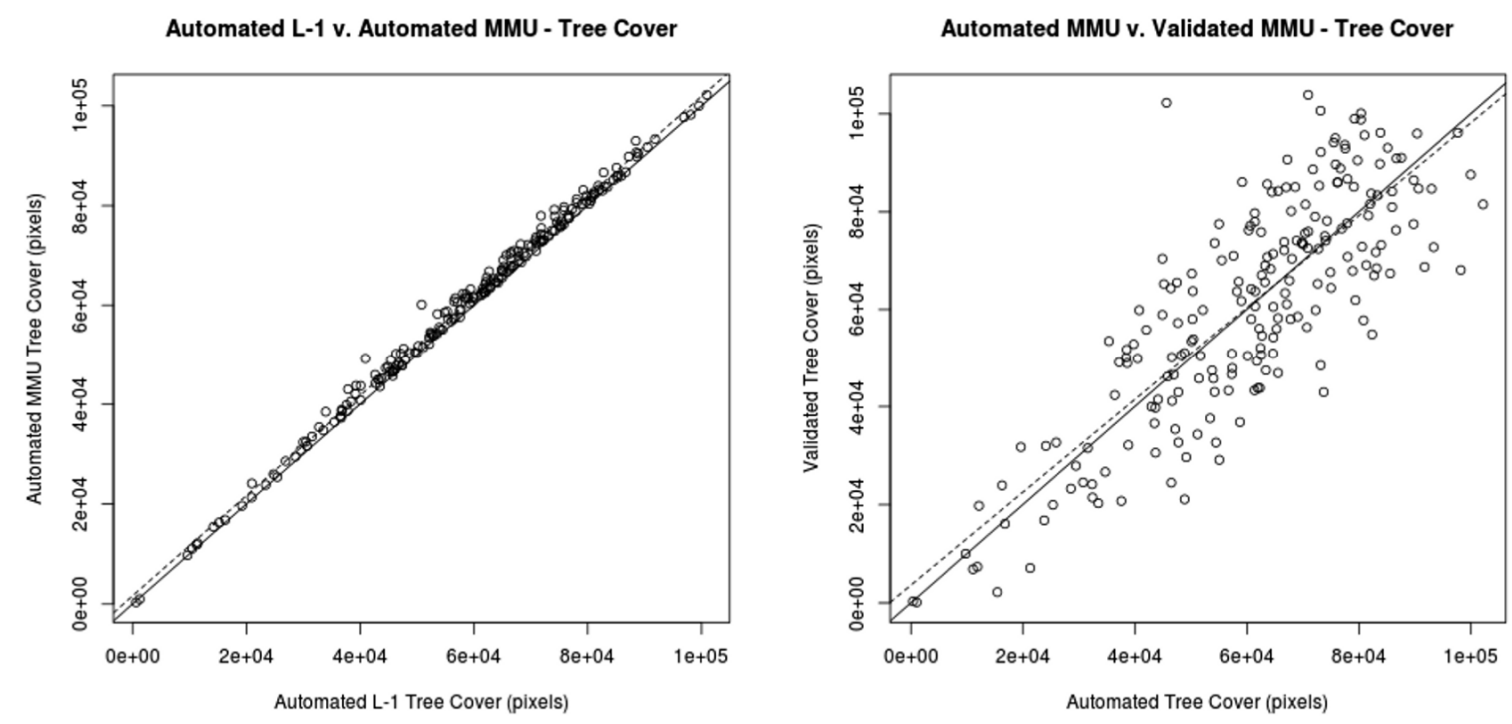

Figure 4. (Left) A comparison of the tree cover area obtained automatically for level-1 segmentation (x-axis) and the 5 ha MMU (y-axis); and (Right) a comparison of the tree cover area obtained automatically at the 5 ha MMU (x-axis) and the expert reviewed-revised tree cover at the 5 ha MMU (y-axis). The dotted line represents the slope of the line formed by the linear regression. The solid line represents the one-to-one line.

\subsection{OBIA, Minimum Mapping Units and Change Detection}

To analyse the effects of increasing segment size on amount of area detected as change, an automated, per-pixel change detection was performed for a sub-sample of sites employing the IR-MAD algorithm and the Landsat samples from 1990 and 2000. A series of nine change likelihood thresholds in one per cent increments, ranging from 91\% to 99\% [43,47], were used in the iterations. Pixels with change likelihoods above the threshold in each run were labelled as change, all other pixels were labelled as no-change (Figure 5 top-row). Changed pixels were then aggregated by level-1 and 5 ha MMU segments using a simple majority rule (i.e., if $>50 \%$ of a segment contained change, the segment was labelled as change) (Figure 5, middle and bottom row, respectively). The difference in area changed for each iteration of the IR-MAD algorithm and for each segmentation level was analysed.

Logically, as the threshold of the IR-MAD algorithm increases and change pixels are more selectively labelled, the mean area of change decreases. The difference in the amount of change detected between the pixel-based and segmentation aggregations also decreases. However, the aggregation to segments results in an overall decrease in the amount of change detected between $25 \%$ and $50 \%$ when compared to the pixel-level analyses (Figure 6, left). 

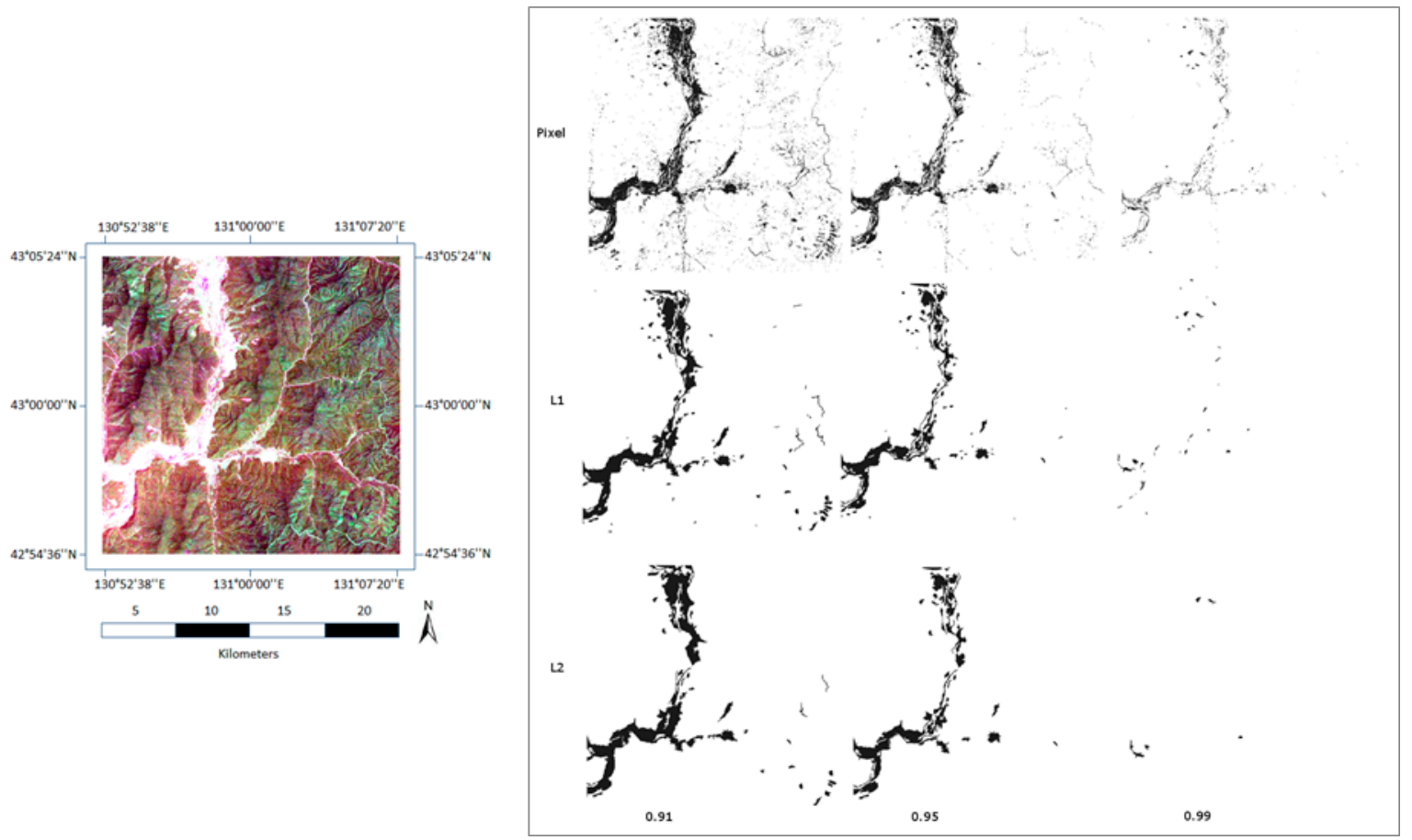

Figure 5. The amount of change detected between 1990-2000 using IR-MAD and increasing change thresholds (columns) aggregated to level- 1 and 5 ha MMU segments (rows) for a $20 \times 20 \mathrm{~km}$ sample site. Columns left to right represent thresholds of $0.91,0.95$ and 0.99 of the IR-MAD change likelihood layer, respectively. Rows top to bottom represent pixel-level, level-1 image segments and 5 ha MMU segments, respectively. Changed areas are in black. Non-changed areas are white.

\subsection{Landscape Metrics and Change Detection}

The difference in area change per level of aggregation could be explained by the spatial arrangement and areal extent of the detected change in each of the iterations. Landscape metrics were generated for the pixel-based change layer using the R statistical package SDMTools [48]. Landscape metrics are algorithms that describe the spatial characteristics and pattern of land cover classes on a landscape and are commonly applied in ecological studies [49]. Riiters et al. [50] emphasized that landscape metrics must be cautiously applied as many are co-variates and suggested six main landscape metrics that are the most suitable for describing landscapes with real implications.

To determine if the size and shape of areas detected as change had an effect on the amount and type of change detected in the image segments, we used two of Riiters et al. [50] recommended landscape metrics, mean patch size and mean landscape shape index, and calculated these based on the pixel-level change detection. Then, we compared the difference in area between change detection iterations and aggregations as a function of each of the calculated metrics. Mean patch size is the mean area of all change patches within the sample site. Mean landscape shape index is the relative perimeter-to-area ratio of the landscape (i.e., values close to $1=$ compact, values increase as patches become disaggregated and amount of edge increases). The difference in area detected as change between the iterations and aggregation levels varies inversely with mean change patch area (e.g., as the size of change patch increases, the difference in area detected at aggregated spatial levels decreases) (Figure 6, right). Figure 6 (centre) shows there is a strong linear relationship $\left(R^{2}=0.9\right)$ between increasing landscape shape index and increased differences in the total area of change detected between the pixel-based and object-based estimates, indicating that as change patches become more disaggregated and have a higher perimeter-to-area ratio, they are more difficult to detect in segments meeting the MMU requirement. 

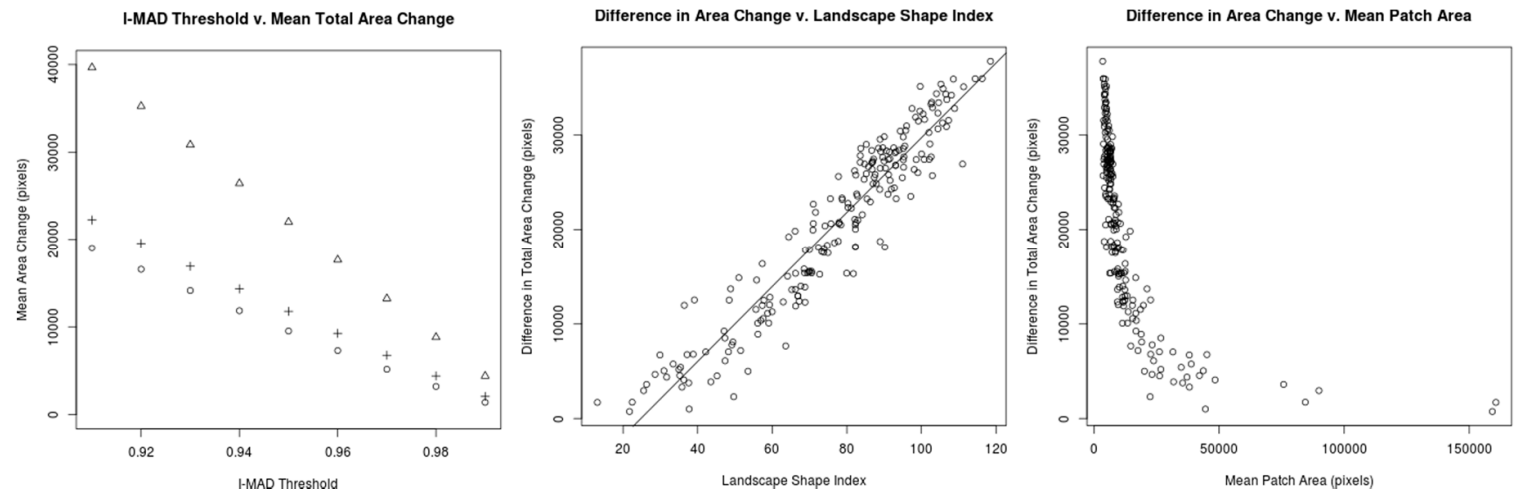

Figure 6. (Left) The mean area detected as change (Y-axis) over all samples using pixel-based (triangles), level-1 segments (+) and level-2 segments (circles) at increasing IR-MAD thresholds (X-axis). (Centre) The difference in the total area detected as change (Y-axis) between pixel and level-2 segments plotted as a function of the change segments' landscape shape index (X-axis). (Right) The difference in the total area detected as change (Y-axis) between pixel and level-2 segments plotted as a function of the mean change patch area (X-axis).

Small or complex landscape features (e.g., areas of change) are more difficult to detect in the 5 ha MMU image segments. Whether or not these potential omissions have significant meaning on the landscape, however, is unknown. It is intuitive that patches of change smaller than the MMU are largely lost when the analysis shifts from pixel-based to segment-based. It is also true, however, that patches of change smaller than the MMU remain detectable at the segment level, indicating that some segments labelled as "change" actually overestimate the actual area changed. The figure shows, then, that the total area of a change patch is not the only indicator of whether it is detectable or not at aggregated spatial scales. There is a strong relationship between the landscape shape index and the difference in total area changed between the iterations. This indicates that the complexity of the patch shape has a high degree of influence on its detectability. More compact shapes (i.e., clear-cut timber harvest) are thus more easily detectable than more complex shapes, which are often characterized by large perimeter to area ratios (i.e., logging roads, selective logging, small-scale agriculture).

\subsection{Automated Land-Cover Classification and Change Detection Results}

To determine the effectiveness of the classification and change detection routine at facilitating the efficient manual review and revision of the samples, the automated results were compared against the expert reviewed and revised land cover labels. For a randomly selected subset of segments, the automated land cover classification achieved an overall agreement of $76 \%$ for all five classes in year 2000 (Table 3). Similar agreements were obtained for years 1990 and 2005.

Table 3. Confusion matrix between land cover classes before and after expert review and revision for a sample of year 2000 sites. Figures are based on area in each class (thousands of hectares). Automatically mapped class labels are on left-hand side and the expert reviewed and revised labels are across the top of the table. Tree $=$ tree cover, Wooded $=$ other wooded land, Other $=$ other land cover.

\begin{tabular}{c|ccccccc}
\hline & Tree & Wooded & Other & Water & No Data & Total & User's \\
\hline Tree & 6957 & 802 & 1599 & 61 & 33 & 9451 & 0.74 \\
Wooded & 564 & 1609 & 2336 & 10 & 2 & 4521 & 0.36 \\
Other & 580 & 793 & 12,684 & 28 & 21 & 14,107 & 0.90 \\
Water & 17 & 24 & 92 & 705 & 3 & 840 & 0.84 \\
No Data & 1 & 3 & 40 & 2 & 103 & 149 & 0.69 \\
Total & 8120 & 3230 & 16,751 & 805 & 162 & 29,069 & \\
Producer's & 0.86 & 0.50 & 0.76 & 0.87 & 0.63 & & $\mathbf{0 . 7 6}$ \\
\hline
\end{tabular}


The automated change/no-change mapping achieved an overall agreement of $88 \%$ between 1990 and 2000 and $87 \%$ between 2000 and 2005 compared to the expert reviewed and revised classification. The relatively high overall agreement is due to the dominance of unchanging classes. Producer's agreement for actual change classes ranged from $68 \%$ to $77 \%$ in both comparisons. User's agreement for actual change classes ranged from $11 \%$ to $36 \%$.

Table 4 shows the confusion matrix generated for each survey period by comparing the results of the automated classification (original) and the expert reviewed and revised land cover dataset (validated).

Table 4. Confusion matrix for change classes for the time periods 1990-2000 (top) and 2000-2005 (bottom) before and after expert review and revision. Figures are based on area in each class (thousands of hectares). Automatically mapped class labels are on left-hand side and the expert reviewed and revised labels are across the top of the table. Tree-Tree $=$ Tree cover in both periods, Tree-Other $=$ Tree cover change to other land, Other-Tree = other land to tree cover, Other-Other = other land cover in both periods.

\begin{tabular}{|c|c|c|c|c|c|c|}
\hline 1990-2000 & Tree-Tree & Tree-Other & Other-Tree & Other-Other & Total & User's \\
\hline Tree-Tree & 4447 & 63 & 33 & 1391 & 5934 & 0.75 \\
\hline Tree-Other & 107 & 211 & 2 & 602 & 922 & 0.23 \\
\hline Other-Tree & 117 & 1 & 138 & 372 & 628 & 0.22 \\
\hline Other-Other & 214 & 28 & 17 & 16,014 & 16,273 & 0.98 \\
\hline Total & 4885 & 303 & 190 & 18,379 & 23,757 & \\
\hline Producer's & 0.91 & 0.70 & 0.73 & 0.87 & & 0.88 \\
\hline 2000-2005 & Tree-Tree & Tree-Other & Other-Tree & Other-Other & Total & User's \\
\hline Tree-Tree & 4185 & 49 & 37 & 1449 & 5720 & 0.73 \\
\hline Tree-Other & 69 & 226 & 1 & 327 & 623 & 0.36 \\
\hline Other-Tree & 119 & 2 & 100 & 665 & 886 & 0.11 \\
\hline Other-Other & 182 & 19 & 9 & 15,393 & 15,603 & 0.99 \\
\hline Total & 4555 & 296 & 147 & 17,834 & 22,832 & \\
\hline Producer's & 0.92 & 0.76 & 0.68 & 0.86 & & 0.87 \\
\hline
\end{tabular}

Land cover and land-use change are statistically "rare" phenomena [51] and, as discussed previously, can be difficult to characterize. However, the low levels of agreement found in the study are lower than desired for the automated classification routine, which in all cases overestimated the amount of change. For a sub-sample of FAO-processed sites in boreal, temperate and subtropical regions, automated results overestimated tree cover loss by $11 \%$ and tree cover gain by $13.5 \%$ compared to the final expert reviewed and revised results for the time period from 1990 to 2005. The lowest user's agreement was consistently found in the "other land to forest" category suggesting that forest re-growth was the most difficult land cover change to accurately detect.

The consistent overestimation of tree cover change in this study is likely caused by several factors including the tendency of the classification rules to favour tree cover over other land cover classes and a rather liberal threshold of the IR-MAD variable used to indicate whether or not an image object represented land cover change. The results clearly suggest that a much stricter rule can safely be applied to flag potentially changed image objects in future iterations of the survey. It may also be advisable, in areas where finer-scale changes are known to occur or are suspected, to selectively ease or eliminate the minimum mapping unit restriction in order to avoid over or under-estimating change.

\subsection{Effect of Land Cover or Land Use Definition on Forest Area Calculations}

We examined the mean proportion of tree cover and forest land use by continent/country and climatic domain for year 2000 in order to assess the effect of using a land cover or land use definition on the calculation of "forest" area (Table 5). For many continental/climate groups, the difference between land cover and land use are minimal. However, for other continental/climate groups, there are 
differences between land cover and land use that, depending on which one is used to calculate "forest" area, could have an impact on global figures.

Table 5. The proportion of global continent/country groupings by climatic domain in forest land use and tree cover in year 2000.

\begin{tabular}{cccc}
\hline Continent/Country & Climatic Domain & Forest Land-Use (Proportion) & Tree Cover (Proportion) \\
\hline Canada & boreal & 0.72 & 0.69 \\
Europe & boreal & 0.69 & 0.69 \\
Africa & subtropical & 0.04 & 0.04 \\
Europe (ex. Russian Fed.) & subtropical & 0.20 & 0.20 \\
North and Central America & subtropical & 0.23 & 0.25 \\
Oceania & subtropical & 0.07 & 0.07 \\
South America & subtropical & 0.11 & 0.11 \\
Asia & temperate & 0.63 & 0.61 \\
Canada & temperate & 0.62 & 0.59 \\
Europe (ex. Russian Fed.) & temperate & 0.33 & 0.33 \\
North and Central America & temperate & 0.29 & 0.32 \\
Oceania & temperate & 0.51 & 0.51 \\
South America & temperate & 0.16 & 0.16 \\
Africa & tropical & 0.20 & 0.16 \\
Asia & tropical & 0.25 & 0.27 \\
Oceania & tropical & 0.46 & 0.46 \\
South America & tropical & 0.18 & 0.18 \\
\hline
\end{tabular}

North and Central America (excluding Canada), in both the subtropical and temperate climatic domain, show a $5 \%$ higher proportion of tree cover than forest land-use. This is probably due to the extensive area of tree cover located in urban settings within the United States [52]. Agroforestry in Central America may also contribute to this difference [53].

Tropical Asia also exhibits a higher proportion of tree cover than forest land-use ( $2 \%)$. This is likely an illustration of the effect of palm oil plantations that are classified as tree cover from a biophysical standpoint but do not meet the requirement for forest land-use [54].

Conversely, boreal and temperate zones of Canada indicate $6 \%$ more forest land-use than detected tree cover. This is most probably due to the large amount of forest burned and logged annually within the boreal and temperate zones of Canada [55]. Though these areas may be classified from a biophysical standpoint as devoid of tree cover and non-forest at the time of the satellite image acquisition, tree cover will regenerate and the areas remain considered as forest land-use.

Temperate Asia shows 2\% more forest land-use than tree cover. This is likely due to the large areas designated as tree plantations in China [56] that may not be fully planted or fully matured to the point of being detectable by a moderate spatial resolution sensor such as Landsat.

Interestingly, tropical Africa indicates $4 \%$ more forest land-use than tree cover. We hypothesize that this may be due to the visual inspection process by local, national experts who could more accurately identify a site as forest in areas where tree cover was sparse and not adequately detected by the medium spatial resolution Landsat sensor [1].

\section{Conclusions}

The OBIA classification and change detection methodology described in this paper provided an efficient means of processing over 11,000 sample sites, 33,000 Landsat $20 \times 20 \mathrm{~km}$ sample tiles and more than 6.5 million individual polygons over three epochs. Comparisons of automated classification results with expert-corrected results yielded agreements of approximately $80 \%$. The automated land cover classification methods thus provided suitable results and decreased the amount of time necessary for expert review and revision. 
Implementing a relatively large MMU (5 ha) facilitated expert human interpretation, review and revision of the results and overcame issues related to fine-scale spectral heterogeneity. However, if the local change dynamic is very fine-scale, a large MMU applied to medium spatial resolution imagery may be incapable of adequately capturing this. In these areas, a strict adherence to a MMU may not be helpful and employing a much smaller or no MMU is advisable.

While pixel-based classification methods may indeed suffer from the "salt-and-pepper" effect of individual pixels being miss-classified [18], image segmentation with a MMU may suffer a similarly confounding fate by systematically under-segmenting areas of land cover change, some or all of which may be ecologically significant.

Finally, land use classifications provide a critical but largely absent component of current land surface change studies. They provide the only information that can elucidate drivers of forest cover change and, subsequently, suggest pathways for land managers seeking to promote or prevent such change. The conversion of land cover to land use, and subsequent expert human review and revision in this study enabled differentiation of relatively temporary changes in the biophysical properties of the land surface from longer-term permanent land use conversions. This has important implications for determining net forest area change, not only gross area losses and may help to explain some of the differences in current estimates of global "forest" area and change.

Acknowledgments: The authors would like to thank the research team at the JRC including Frederic Achard, Hans-Jurgen Stibig, Catherine Bodart, René Beuchle, Andreas Brink, Silvia Carboni, François Donnay, Hugh D. Eva, Desirée Johansson, Ouns Kissiyar, Andrea Lupi, Philippe Mayaux, Rastislav Raši, Dario Simonetti, Hans-Jürgen Stibig, Michael Vollmar, Roman Seliger, Jesús San-Miguel-Ayanz, Pieter Kempeneers, Fernando Sedano, Lucia Seebach, Peter Strobl, and Peter Vogt. The authors also wish to thank the national forestry experts who assisted with the review and revision of the RSS sample sites (http://www.fao.org/ forestry/fra/67944/en/). The authors also wish to thank 4 anonymous reviewers whose comments improved the manuscript. Funding for the FAO Remote Sensing Survey was provided by the European Commission.

Author Contributions: E.L. and R.D. conceived and designed the experiments; E.L. and R.D. performed the experiments; E.L and R.D. analyzed the data; E.L. and R.D. wrote the paper.

Conflicts of Interest: The authors declare no conflict of interest.

\section{Abbreviations}

The following abbreviations are used in this manuscript:

$\begin{array}{ll}\text { OBIA } & \text { Object-based Image Analysis } \\ \text { FAO } & \text { United Nations Food and Agriculture Organization } \\ \text { JRC } & \text { Joint Research Centre of the European Commissions } \\ \text { USGS } & \text { United States Geological Survey } \\ \text { GLS } & \text { Global Land Survey } \\ \text { MMU } & \text { Minimum Mapping Unit } \\ \text { IR-MAD } & \text { Iteratively Re-weighted Multivariate Alteration Detection } \\ \text { MODIS } & \text { Moderate Resolution Imaging Spectroradiometer } \\ \text { ANN } & \text { Artificial Neural Network } \\ \text { REDD } & \text { Reducing Emissions from Deforestation and Degradation }\end{array}$

\section{References}

1. Lindquist, E.J.; D'Annunzio, R.; Gerrand, A.; MacDicken, K.; Achard, F.; Beuchle, R.; Stibig, H.-J. FAO Forestry Paper 169: Global Forest Land-Use Change from 1990-2005; Food and Agriculture Organization of the United Nations: Rome, Italy, 2012. Available online: http://www.fao.org/docrep/017/i3110e/i3110e.pdf (accessed on 18 August 2016).

2. Blaschke, T.; Hay, G.J.; Kelly, M.; Lang, S.; Hofmann, P.; Addink, E.; Tiede, D. Geographic Object-Based Image Analysis-Towards a new paradigm. ISPRS J. Photogramm. Remote Sens. 2014, 87, 180-191. [CrossRef] [PubMed]

3. Blaschke, T. Object based image analysis for remote sensing. ISPRS J. Photogramm. Remote Sens. 2010, 65, 2-16. [CrossRef] 
4. Ball, G.H.; Hall, D.J. ISODATA, An Novel Method of Data Analysis and Pattern Classification; Stanford Research Institute: Menlo Park, CA, USA, 1965; p. 79.

5. MacQueen, J. Some methods for classification and analysis of multivariate observations. Proc. Fifth Berkeley Symp. Math. Statist. Prob. 1967, 1, 281-297.

6. Hussain, M.; Chen, D.; Cheng, A.; Wei, H.; Stanley, D. Change detection from remotely sensed images: From pixel-based to object-based approaches. ISPRS J. Photogramm. Remote Sens. 2013, 80, 91-106. [CrossRef]

7. Vieira, M.A.; Formaggio, A.R.; Rennó, C.D.; Atzberger, C.; Aguiar, D.A.; Mello, M.P. Object Based Image Analysis and data mining applied to a remotely sensed Landsat time-series to map sugarcane over large areas. Remote Sens. Environ. 2012, 123, 553-562. [CrossRef]

8. Eva, H.; Carboni, S.; Achard, F.; Stach, N.; Durieux, L.; Faure, J.-F.; Mollicone, D. Monitoring forest areas from continental to territorial levels using a sample of medium spatial resolution satellite imagery. ISPRS J. Photogramm. Remote Sens. 2010, 65, 191-197. [CrossRef]

9. Eva, H.D.; Achard, F.; Beuchle, R.; de Miranda, E.; Carboni, S.; Seliger, R.; Vollmar, M.; Holler, W.A.; Oshiro, O.T.; Barrena Arroyo, V.; et al. Forest cover changes in Tropical South and Central America from 1990 to 2005 and related carbon emissions and removals. Remote Sens. 2012, 4, 1369-1391. [CrossRef]

10. Duveiller, G.; Defourny, P.; Desclee, B.; Mayaux, P. Deforestation in Central Africa: Estimates at regional, national and landscape levels by advanced processing of systematically-distributed Landsat extracts. Remote Sens. Environ. 2008, 112, 1969-1981. [CrossRef]

11. Ernst, C.; Verhegghen, A.; Bodart, C.; Mayaux, P.; De Wasseige, C.; Bararwandika, A.; Begoto, G.; Esono Mba, F.; Ibara, M.; Kondjo Shoko, A.; et al. Congo Basin forest cover change estimate for 1990, 2000 and 2005 by Landsat interpretation using an automated object-based processing chain. Int. Arch. Photogramm. Remote Sens. Spat. Inf. Sci. 2010, 38, 1-6.

12. Mayaux, P.; Pekel, J.-F.; Desclée, B.; Donnay, F.; Lupi, A.; Achard, F.; Clerici, M.; Bodart, C.; Brink, A.; Nasi, R.; et al. State and evolution of the African rainforests between 1990 and 2010. Phil. Trans. R. Soc. B 2013, 368. [CrossRef] [PubMed]

13. Raši, R.; Bodart, C.; Stibig, H.J.; Eva, H.; Beuchle, R.; Carboni, S.; Simonetti, D.; Achard, F. An automated approach for segmenting and classifying a large sample of multi-date Landsat imagery for pan-tropical forest monitoring. Remote Sens. Environ. 2011, 115, 3659-3669. [CrossRef]

14. Brink, A.B.; Eva, H.D. Monitoring 25 years of land cover change dynamics in Africa: A sample based remote sensing approach. Appl. Geogr. 2009, 29, 501-512. [CrossRef]

15. Bodart, C.; Brink, A.B.; Donnay, F.; Lupi, A.; Mayaux, P.; Achard, F. Continental estimates of forest cover and forest cover changes in the dry ecosystems of Africa between 1990 and 2000. J. Biogeogr. 2013, 40, 1036-1047. [CrossRef] [PubMed]

16. Woodcock, C.; Strahler, A. The factor of scale in remote sensing. Remote Sens. Environ. 1987, 21, 311-332. [CrossRef]

17. Marceau, D.J.; Hay, G.J. Remote sensing contributions to the scale issue. Can. J. Remote Sens. 1999, 25, 357-366. [CrossRef]

18. Yu, Q.; Gong, P.; Clinton, N.; Biging, G.; Kelly, M.; Schirokauer, D. Object-based detailed vegetation classification with airborne high spatial resolution remote sensing imagery. Photogramm. Eng. Remote Sens. 2006, 72, 799-811. [CrossRef]

19. Gutman, B.G.; Byrnes, R.; Masek, J.; Covington, S.; Justice, C.; Franks, S.; Headley, R. Towards monitoring land-cover and land-use changes at a global scale: The Global Land Survey 2005. Photogramm. Eng. Remote Sens. 2008, 74, 6-10.

20. Toivonen, T.; Kalliola, R.; Ruokolainen, K.; Naseemmalik, R. Across-path DN gradient in Landsat TM imagery of Amazonian forests: A challenge for image interpretation and mosaicking. Remote Sens. Environ. 2006, 100, 550-562. [CrossRef]

21. Potapov, P.; Hansen, M.C.; Gerrand, A.M.; Lindquist, E.J.; Pittman, K.; Turubanova, S.; Wilkie, M.L. The global Landsat imagery database for the FAO FRA remote sensing survey. Int. J. Digit. Earth 2011, 4, 2-21. [CrossRef]

22. Bodart, C.; Eva, H.; Beuchle, R.; Raši, R.; Simonetti, D.; Stibig, H.-J.; Brink, A.; Lindquist, E.; Achard, F. Pre-processing of a sample of multi-scene and multi-date Landsat imagery used to monitor forest cover changes over the tropics. ISPRS J. Photogramm. Remote Sens. 2011, 66, 555-563. [CrossRef]

23. Beuchle, R.; Eva, H.D.; Stibig, H.J.; Brink, A.; Mayaux, P.; Achard, F.; Belward, A. A satellite data set for tropical forest area change assessment. Int. J. Remote Sens. 2011, 32, 7009-7031. [CrossRef] 
24. Achard, F.; Stibig, H.J.; Eva, H.; Mayaux, P.; Va, I.-I. Tropical forest cover monitoring in the humid tropics-TREES project. Trop. Ecol. 2002, 43, 9-20.

25. JRC. “FORESTMOD Action Website". 2012. Available online: http://forest.jrc.ec.europa.eu/ (accessed on 9 December 2015).

26. Baatz, M.; Schäpe, A. Multiresolution segmentation: an optimization approach for high quality multi-scale image segmentation. In Angewandte Geographische Informations-Verarbeitung XII; Strobl, J., Blaschke, T., Griesbner, G., Eds.; Wichmann Verlag: Karlsruhe, Germany, 2000; pp. 12-23.

27. Desclee, B.; Bogaert, P.; Defourny, P. Forest change detection by statistical object-based method. Remote Sens. Environ. 2006, 102, 1-11. [CrossRef]

28. Mesner, N.; Oštir, K. Investigating the impact of spatial and spectral resolution of satellite images on segmentation quality. J. Appl. Remote Sens. 2014, 8, 083696. [CrossRef]

29. Ridder, R. Global Forest Resources Assessment 2010: Options and Recommendations for a Global Remote Sensing Survey of Forests. FAO Forestry Working Paper 141. 2007. Available online: ftp://ftp.fao.org/docrep/fao/ 010/ai074e/ai074e00.pdf (accessed on 18 August 2016).

30. Horler, D.N.H.; Ahern, F.J. Forestry information content of Thematic Mapper data. Int. J. Remote Sens. 1986, 7, 405-428. [CrossRef]

31. Hoffhine, E.; Sader, S.A. Detection of forest harvest type using multiple dates of Landsat TM imagery. Remote Sens. Environ. 2002, 80, 385-396.

32. Hansen, M.C. Global percent tree cover at a spatial resolution of 500 meters: First results of the MODIS vegetation continuous fields algorithm. Earth Interact. 2003, 7. [CrossRef]

33. Arino, O.; Gross, D.; Ranera, F.; Leroy, M.; Bicheron, P.; Brockman, C.; Defourny, P.; Vancutsem, C.; Achard, F.; Durieux, L.; et al. GlobCover: ESA service for Global land cover from MERIS. In Proceedings of the IEEE International Geoscience and Remote Sensing Symposium, 2007 (IGARSS 2007), Barcelona, Spain, 23-28 July 2007; pp. 2412-2415.

34. Carroll, M.L.; Townshend, J.R.; DiMiceli, C.M.; Noojipady, P.; Sohlberg, R.A. A new global raster water mask at $250 \mathrm{~m}$ resolution. Int. J. Dig. Earth 2009, 2, 291-308. [CrossRef]

35. Yuan, H.; Van Der Wiele, C.F.; Khorram, S. An Automated Artificial Neural Network System for land use/land cover classification from Landsat TM Imagery. Remote Sens. 2009, 1, 243-265. [CrossRef]

36. Mas, J.F.; Flores, J.J. The application of artificial neural networks to the analysis of remotely sensed data. Int. J. Remote Sens. 2008, 29, 617-663. [CrossRef]

37. Foody, G.M.; Arora, M.K. An evaluation of some factors affecting the accuracy of classification by an artificial neural network. Int. J. Remote Sens. 1997, 18, 799-810. [CrossRef]

38. Bachmann, F. Neural Network Plugin for Definiens Developer 7.0 Short Reference; User Manual; Technische Universitat Bergakademie Freiberg: Freiberg, Germany, 2009; Available online: http://www. treatymonitoring.de/tools/ShortReference_NN.pdf (accessed on 18 August 2016).

39. Hansen, M.; Roy, D.; Lindquist, E.; Adusei, B.; Justice, C.; Altstatt, A. A method for integrating MODIS and Landsat data for systematic monitoring of forest cover and change in the Congo Basin. Remote Sens. Environ. 2008, 112, 2495-2513. [CrossRef]

40. Foody, G.M. Mapping land cover from remotely sensed data with a Softened Feedforward Neural Network Classification. J. Intell. Robot. Syst. 2000, 29, 433-449. [CrossRef]

41. Nielsen, A.A.; Conradsen, K.; Simpson, J.J. Multivariate Alteration Detection (MAD) and MAF postprocessing in multispectral, bitemporal image data: New approaches to change detection studies. Remote Sens. Environ. 1998, 64, 1-19. [CrossRef]

42. Nielsen, A.A. The regularized iteratively reweighted MAD method for change detection in multi- and hyperspectral data. IEEE Trans. Image Process. 2007, 16, 463-478. [CrossRef] [PubMed]

43. Nielsen, A.A. An iterative extension to the MAD transformation for change detection in multi- and hyperspectral remote sensing data. Math. Model. 2005, 11, 27-29.

44. John, A.; Bachman, F. MAD-Transformation Plugin; User Manual; Technische Universitat Bergakademie Freiberg: Freiberg, Germany, 2009. Available online: http://www.treatymonitoring.de/tools/mad_manual. pdf (accessed on 18 August 2016).

45. Lambin, E.; Turner, B.; Geist, H.; Agbola, S.; Angelsen, A.; Bruce, J.; Coomes, O.T.; Dirzo, R.; Fischer, G.; Folke, C.; et al. The causes of land-use and land-cover change: Moving beyond the myths. Glob. Environ. Chang. 2001, 11, 261-269. [CrossRef] 
46. Coulston, J.W.; Reams, G.A.; Wear, D.N.; Brewer, C.K. An analysis of forest land use, forest land cover and change at policy-relevant scales. Forestry 2013, 87, 267-276. [CrossRef]

47. Canty, M.J.; Nielsen, A.A. Visualization and unsupervised classification of changes in multispectral satellite imagery. Int. J. Remote Sens. 2006, 27, 3961-3975. [CrossRef]

48. VanDerWal, J.; Falconi, L.; Januchowski, S.; Shoo, L.; Storlie, C. SDMTools: Species Distribution Modelling Tools: Tools for Processing Data Associated with Species Distribution Modelling Exercises; R Package Version; 2011.

49. McGarigal, K.; Marks, B.J. Spatial Pattern Analysis Program for Quantifying Landscape Structure; Gen. Tech. Rep. PNW-GTR-351; US Department of Agriculture, Forest Service, Pacific Northwest Research Station: Portland, OR, USA, 1995.

50. Riitters, K.H.; O'neill, R.V.; Hunsaker, C.T.; Wickham, J.D.; Yankee, D.H.; Timmins, S.P.; Jones, K.B.; Jackson, B.L. A factor analysis of landscape pattern and structure metrics. Landsc. Ecol. 1995, 10, 23-39. [CrossRef]

51. Stehman, S.V.; Sohl, T.L.; Loveland, T.R. An evaluation of sampling strategies to improve precision of estimates of gross change in land use and land cover. Int. J. Remote Sens. 2005, 26, 4941-4957. [CrossRef]

52. Nowak, D.J.; Greenfield, E.J.; Hoehn, R.E.; Lapoint, E. Carbon storage and sequestration by trees in urban and community areas of the United States. Environ. Pollut. 2013, 178, 229-236. [CrossRef] [PubMed]

53. Garrity, D. Agroforestry and the future of global land use. In Agroforestry - The Future of Global Land Use; Nair, P.K.R., Garrity, D., Eds.; Springer Netherlands: Dordrecht, The Netherlands, 2012.

54. Carlson, K.M.; Curran, L.M.; Asner, G.P.; Pittman, A.M.; Trigg, S.N.; Adeney, J.M. Carbon emissions from forest conversion by Kalimantan oil palm plantations. Nat. Clim. Chang. 2013, 3, 283-287. [CrossRef]

55. Natural Resources Canada. The State of Canada's Forests: Annual Report 2014; Natural Resources Canada, Canadian Forest Service: Ottawa, ON, Canada, 2014.

56. Liu, M.; Tian, H. China's land cover and land use change from 1700 to 2005: Estimations from high-resolution satellite data and historical archives. Glob. Biogeochem. Cycles 2010, 24. [CrossRef]

(C) 2016 by the authors; licensee MDPI, Basel, Switzerland. This article is an open access article distributed under the terms and conditions of the Creative Commons Attribution (CC-BY) license (http:/ / creativecommons.org/licenses/by/4.0/). 